\title{
Estudio de la microestructura de un cordón de soldadura de un acero P91 mediante microscopía electrónica de transmisión
}

\section{Study of the microstructure of a weld bead P91 steel by transmission electron microscopy}

\author{
Ana Lucía Marzocca ${ }^{1}$, Flavio Soldera ${ }^{2}$
} Mónica Zalazar ${ }^{3}$, María Inés Luppo ${ }^{1}$

\author{
${ }^{1}$ Gerencia Materiales, Comisión Nacional de Energía Atómica, Av. G. Paz 1499, San Martín, Buenos Aires, Argentina. \\ e-mail: marzocca@cnea.gov.ar, luppo@cnea.gov.ar \\ ${ }^{2}$ Department of Materials Science, Saarland University, D-66123 Saarbrücken, Sarre, Alemania. \\ e-mail: f.soldera@matsci.uni-sb.de \\ ${ }^{3}$ Laboratorio de Metalografía y de Soldadura, Universidad Nacional Del Comahue, Buenos Aires 1400, Patagônia, Neu- \\ quén, Argentina. \\ e-mail: monica.zalazar@fain.uncoma.edu
}

\section{RESUMEN}

En el presente trabajo se llevó a cabo la caracterización microestructural de un cordón de pasada simple de un acero $9 \mathrm{Cr} 1 \mathrm{MoVNb}$ P91 obtenido por el proceso de soldadura semiautomática bajo protección gaseosa (FCAW), en la condición "as welding” mediante microscopía electrónica de transmisión.

El acero P91 tiene una buena soldabilidad, pero las propiedades mecánicas de las uniones soldadas son inferiores a las del material base. La zona afectada por el calor (ZAC) de este tipo de aceros es de $\sim 4 \mathrm{~mm}$ lo que dificulta extraer réplicas y láminas delgadas para el estudio de las distintas microestructuras generadas en cada sub-zona de la ZAC: ZAC de grano grueso (ZACGG), ZAC de grano fino (ZACGF) y ZAC intercrítica (ZACIC). No obstante, fue posible extraer réplicas de cada región para identificar a los precipitados y por medio de la utilización de un haz de iones focalizados (FIB-SEM) se pudieron extraer láminas delgadas de un área específica para caracterizar la microestructura de la matriz.

Se caracterizaron los precipitados presentes en cada sub-zona de la $\mathrm{ZAC}$ y cómo la disolución del $\mathrm{M}_{23} \mathrm{C}_{6}$ afecta a la matriz que lo rodea. A bajas temperaturas pico y bajas velocidades de calentamiento/enfriamiento tales como las que caracterizan a las zonas de grano fino (ZACIC y ZACGF) se encontró austenita retenida en contacto de los carburos $\mathrm{M}_{23} \mathrm{C}_{6}$ parcialmente disueltos. Por otra parte, en las zonas con más altas temperaturas pico y altas velocidades de calentamiento/enfriamiento (ZACGG y zona fundida) se encontraron películas finas de austenita retenida en los ex bordes de grano austenítico y en los listones de martensita.

Palabras clave: acero $9 \mathrm{Cr} 1 \mathrm{Mo}$, cordón de soldadura, microscopía electrónica.

\section{ABSTRACT}

In the present work the microstructural characterization of a single-pass weld of $9 \mathrm{Cr} 1 \mathrm{MoVNb}$ P91 steel performed by the flux-cored arc welding (FCAW) process in the "as welding" condition has been carried out by means of transmission electron microscopy.

P91 steel offers a good weldability, but the mechanical properties of the welded joints are found to be inferior compared to the base metal. The heat affected zone (HAZ) of these types of steels is $\sim 4$ mm making it difficult to extract carbon replicas and thin foil to study the microstructure generated in each sub-zone of the ZAC: coarse grained ZAC (CGZAC), fine grained ZAC (FGZAC) and intercritical ZAC (ICZAC). Nevertheless, it was possible to extract replicas from each region to identify precipitates and using a focused ion beam (FIB-SEM), thin films could be extracted from a specific area to characterize the matrix microstructure.

The precipitates present in each subzone of the ZAC were characterized as well as how the dissolution of $\mathrm{M}_{23} \mathrm{C}_{6}$ affects the matrix. At low peak temperatures and low heating / cooling rates such as those characterizing the fine grain zones ( $\mathrm{ICHAZ}$ and FGHAZ) the $\mathrm{M}_{23} \mathrm{C}_{6}$ carbides were found to be partially dissolved and in contact with retained austenite. On the other hand, in the areas with the highest peak temperatures and high 
heating/cooling rates (CGHAZ and fusion zone) thin films of retained austenite were found on the austenitic grain boundaries and on the martensítica lath. On the other hand, in the areas with the highest peak temperatures and high heating / cooling rates (ZACGG and ZF) thin films of retained austenite on the austenitic grain edges and the martensite laths were found.

Keywords: 9Cr1Mo steel, Welded joint, Electron microscopy.

\section{INTRODUCCIÓN}

Los aceros 9Cr1MoNbV, pertenecientes al grupo de aceros 9-12\% Cr F/M (ferrítico/martensíticos), se emplean en la fabricación de componentes sometidos a altas temperaturas en centrales térmicas, debido a su excelente resistencia al creep y a la oxidación a alta temperatura [1-3]. A su vez son considerados como los materiales de referencia para los componentes del sistema primario de los futuros reactores nucleares de la llamada Generación IV. La razón de esta preferencia es que poseen mejores propiedades térmicas (mayor conductividad y menor expansión), mejor estabilidad dimensional bajo irradiación y mejor compatibilidad con los metales líquidos que los aceros austeníticos [4].

Para la fabricación de los componentes ya mencionados se necesita del proceso de soldadura. A pesar de que los aceros P91 presentan una buena soldabilidad, las propiedades mecánicas de la junta soldada se vuelven inferiores en comparación a las del metal base [5]. En particular, la reducción en la resistencia al creep de las juntas soldadas pueden ser del orden del 40\% comparada con la del metal base [6].

Como resultado del fuerte ciclo térmico causado por el proceso de soldadura, la microestructura original es alterada y se genera una región llamada zona afectada por el calor (ZAC) como se observa en la Figura 1.Los cambios microestructurales que ocurren en la ZAC necesitan ser entendidos ya que influyen en el rendimiento de la junta soldada durante el servicio [5]. La microestructura que se forma en la ZAC es altamente heterogénea debido a las distintas velocidades de calentamiento/enfriamiento experimentadas y a las temperaturas pico alcanzadas a diferentes distancias desde la línea de fusión. Por esta razón, la ZAC a su vez se divide en las siguientes subzonas: zona de grano grueso (ZACGG), zona de grano fino (ZACGF) y zona intercrítica (ZACIC). A pesar de esta división, no se reconoce un límite definido entre las distintas subzonas; se trata más bien de un gradiente continuo desde el material base $(\mathrm{MB})$ y la zona fundida $(\mathrm{ZF})$.

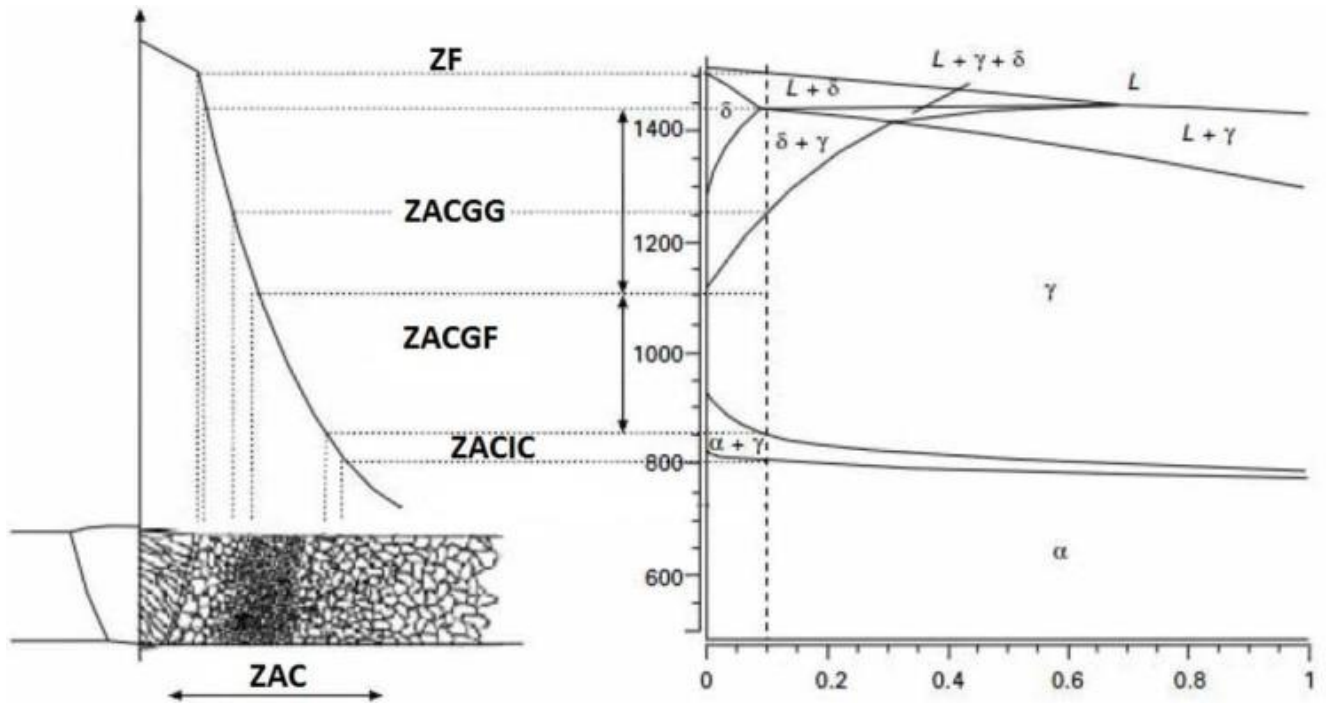

Figura 1: Representación esquemática de la microestructura desarrollada en la ZAC, en función de la temperatura máxima alcanzada durante la soldadura [5].

La mayoría de las fallas en la soldadura de los aceros 9-12\% Cr F/M se presentan en la ZACGF y ZACIC durante el servicio a alta temperatura. Este tipo de falla se conoce como Fisura tipo IV [5]. Realizando ensayos de creep en muestras de un acero 9-12\% Cr F/M (HCM12A) que fueron tratadas a temperaturas entre 800 y $1000{ }^{\circ} \mathrm{C}$ para simular las dos zonas de grano fino, ABE [7] encontró una posible explicación al hecho de que ambas zonas hayan sido asociadas con la falla Tipo IV. De sus resultados obtuvo que a altas tensiones (144 MPa) la falla ocurre preferencialmente en muestras calentadas apenas por debajo de $\mathrm{A}_{\mathrm{c} 3}$, es decir en la ZACIC, mientras que a bajas tensiones (<120 MPa), el mínimo se corrió a las muestras calentadas justo por encima de $\mathrm{A}_{\mathrm{c} 3}$, es decir en la ZACGF.

Uno de las formas básicas de aumentar la resistencia al creep en los aceros 9-12\% Cr F/M es por endu- 
recimiento por precipitación [5]. Estos aceros contienen en su condición de suministro varios tipos de carburos/carbonitruros $\left(\mathrm{M}_{23} \mathrm{C}_{6}\right.$ con $\mathrm{M}=\mathrm{Cr}$, Fe y $\mathrm{MX}$ con $\mathrm{M}=\mathrm{V}, \mathrm{Nb}$ y $\left.\mathrm{X}=\mathrm{C}, \mathrm{N}\right)$ en la matriz y en los bordes de grano como resultado de un adecuado tratamiento térmico que consiste en un normalizado seguido de un revenido. La investigación de estas partículas es de gran importancia para entender el efecto que tienen sobre la resistencia al creep de este tipo de aceros. Por otro lado, estos precipitados se disuelven parcial o totalmente durante el calentamiento/enfriamiento de cada una de las zonas de la ZAC, modificando la matriz que los rodea.

El objetivo de este trabajo fue el de caracterizar las distintas microestructuras introducidas durante la soldadura mediante el proceso FCAW del acero P91 como función del ciclo térmico experimentado por cada región en un cordón de pasada simple en la condición "as welding". Para ello se realizó un estudio detallado por medio de microscopía electrónica de transmisión de réplicas de carbono y láminas delgadas extraídas directamente de cada región de la ZAC del cordón soldado, del MB y de la ZF.

\section{MATERIALES Y MÉTODOS}

Se realizó una soldadura por pasada simple en una cañería de acero ASTM A335 Gr P91, proveniente de la acería japonesa JFE Corporation, con un espesor de pared de $28,5 \mathrm{~mm}$ y $350 \mathrm{~mm}$ de diámetro. El estado metalúrgico de recepción fue: normalizado a $1050{ }^{\circ} \mathrm{C}, 10$ minutos y revenido a $785^{\circ} \mathrm{C}, 45$ minutos. En la Tabla 1 se muestra la composición química del material en la condición de suministro.

Tabla 1: Composición química del acero P91 y del metal de aporte en \%peso.

\begin{tabular}{|c|c|c|c|c|c|c|c|c|c|c|}
\hline \multicolumn{11}{|c|}{ ACERO P91 (WT\%) } \\
\hline $\mathbf{C}$ & Mn & $\mathbf{S i}$ & $\mathbf{N i}$ & $\mathrm{Cr}$ & Mo & Al & $\mathbf{N b}$ & Ti & $\mathbf{V}$ & $\mathbf{N}$ \\
\hline 0,11 & 0,44 & 0,30 & 0,17 & 9,26 & 0,86 & 0,004 & 0,09 & 0,002 & 0,21 & 0,039 \\
\hline \multicolumn{11}{|c|}{ METAL DE APORTE (WT\%) } \\
\hline 0,10 & 0,94 & 0,32 & 0,48 & 9,92 & 0,93 & 0,003 & 0,05 & 0,033 & 0,25 & - \\
\hline
\end{tabular}

El proceso de soldadura se llevó a cabo mediante el proceso FCAW bajo protección gaseosa $80 \% \mathrm{Ar} / 20 \% \mathrm{CO}_{2}$. El metal de aporte fue un alambre tubular AWS A5.29/A5.29M:2010 E91T1, cuya composición química se muestra en la Tabla 1. La temperatura de precalentamiento fue de $260{ }^{\circ} \mathrm{C}$ y los parámetros utilizados para realizar las soldaduras fueron: tensión de trabajo $26 \mathrm{~V}$, corriente $160 \mathrm{~A}$, velocidad de avance 3 $\mathrm{mm} / \mathrm{s}$, lo que generó un calor aportado promedio de $1,4 \mathrm{~kJ} / \mathrm{mm}$.

La preparación metalográfica de las muestras se llevó a cabo puliendo hasta paño con pasta de diamante de $1 \mu \mathrm{m}$ y se realizó el ataque con el reactivo de Villela. Por medio del microscopio óptico Olympus X51 se identificaron cada una de las regiones de la ZAC. La distribución de los precipitados en la matriz y las distintas fases presentes en cada región de la soldadura por pasada simple se observaron en el microscopio electrónico de barrido con cañón por emisión de campo (FEG-SEM) Zeiss Supra 40.

La microscopía electrónica de transmisión se llevó a cabo en un microscopio Philips CM200 (TEM), equipado con un sistema EDAX-DX4 mediante el cual se estudió la composición química de las fases precipitadas por microanálisis de rayos $\mathrm{X}$ dispersivo en energías (EDS).Se examinaron dos tipos de muestras para cada zona: réplicas de carbono, utilizadas para obtener información de la composición química de los precipitados sin interferencia de la matriz martensítica, y láminas delgadas utilizadas para determinar la naturaleza particular de las fases presentes en la matriz. Las láminas delgadas del MB y de la ZF fueron preparadas de manera convencional empleando un TenuPol 5. Para la preparación de las muestras de cada sub-zona de la ZAC se utilizó un microscopio de doble haz FIB-SEM (haz de iones focalizados - microscopio electrónico de barrido) marca FEI modelo Helios 600 con el cual es posible obtener láminas de regiones inaccesibles mediante la técnica convencional, tal como sucede con las distintas sub-zonas dentro de la ZAC. Para extraer las láminas se utilizó la técnica lift-out. El afinado se realizó en el mismo equipo con el haz de iones (Ga) con una tensión de $30 \mathrm{kV}$ y distintas corrientes (400 pA, 200 pA, 93 pA y 48 pA). Para minimizar posible artefactos como amorfización e implantación de $\mathrm{Ga}$, el pulido final fue a $5 \mathrm{kV}$ con una corriente de 16 pA por aproximadamente $2 \mathrm{~min}$.

Los precipitados se identificaron teniendo en cuenta un estudio previo realizado en un acero similar [8] a través de su diagrama de difracción y análisis de EDS: $\mathrm{M}_{23} \mathrm{C}_{6}$ (grupo espacial: 225-Fm-3; parámetro de red, $\mathrm{a}=1,066 \mathrm{~nm} ; 58 \mathrm{Cr}-32,2 \mathrm{Fe}-8,4 \mathrm{Mo}-1,4 \mathrm{~V}$ ); MX tipo I o NbCN (grupo espacial: 225-Fm-3; a = 0,44082 $\mathrm{nm} ; 81,5 \mathrm{Nb}-12,7 \mathrm{~V}-5,8 \mathrm{Cr}$ ); $\mathrm{MX}$ tipo II o VN (grupo espacial: 225-Fm-3; a = 0,41295 nm; 67,1V-11,8Nb21,1Cr/ 57,1V-21,3Nb-19,9Cr); MX tipo III o "wings" (núcleo: NbCN, “alas": VN).

Se realizaron experiencias de difracción de rayos $\mathrm{X}$ sobre la superficie pulida del MB y de la ZF utili- 
zando un Difractómetro Panalytical modelo Empyrean dotado de un tubo de radiación $\mathrm{Cu}-\mathrm{K} \alpha$ y un detector de alta eficiencia PixCel 3D empleando un monocromador de grafito. Las fases presentes en la matriz se identificaron como: austenita (grupo espacial: F m -3 m; parámetro de red, a =0,36013 nm) y martensita (grupo espacial: I m -3 m; parámetro de red, a = 0,28705 nm).

\section{RESULTADOS}

En la Figura 2 se observan las micrografías FEG-SEM de las diferentes zonas de la muestra soldada por pasada simple en las cuales se puede apreciar la distribución de precipitados en la matriz. En las micrografías se señalan las dos fases reconocidas mediante FEG-SEM en la matriz: martensita fresca (MF) y martensita revenida (MR). La coexistencia entre la MF y MR se puede observar en la ZACIC (Figura 2.b.). Las zonas con temperaturas pico $(\mathrm{Tp})$ inferiores a $\mathrm{A}_{\mathrm{c} 1}$ tienen solo $\mathrm{MR}$ ya que estas zonas no alcanzaron las temperaturas necesarias para transformar a austenita durante la soldadura. Las zonas con Tp superiores a $\mathrm{A}_{\mathrm{c} 3}$ (ZACGF, ZACGG y ZF) presentan solo MF dado que estas zonas transformaron completamente a austenita durante el calentamiento en el proceso de soldadura, fase que transforma a martensita con el enfriamiento.
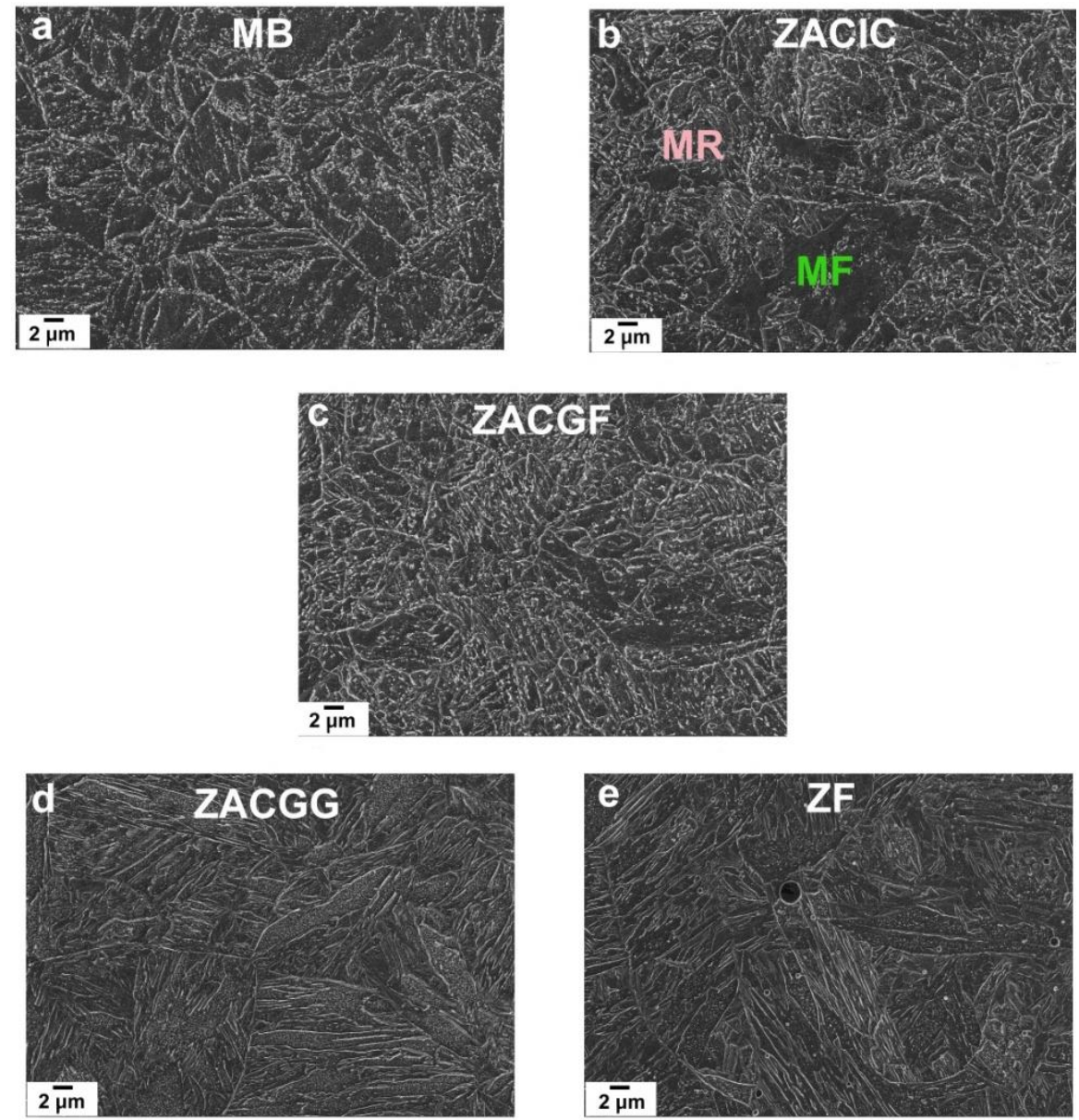

Figura 2: Micrografías FEG-SEM del cordón de soldadura por pasada simple: (a) MB, (b) ZACIC, (c) ZACGF, (d) ZACGG, (e) ZF.

En la Figura 3 se puede observar una vista general de los precipitados encontrados en las distintas zonas de la muestra soldada por pasada simple. En ella se identifican los tipos de partículas por medio de flechas. A medida que se incrementa la Tp, todos los precipitados se van esferoidizando y reduciendo su cantidad. 


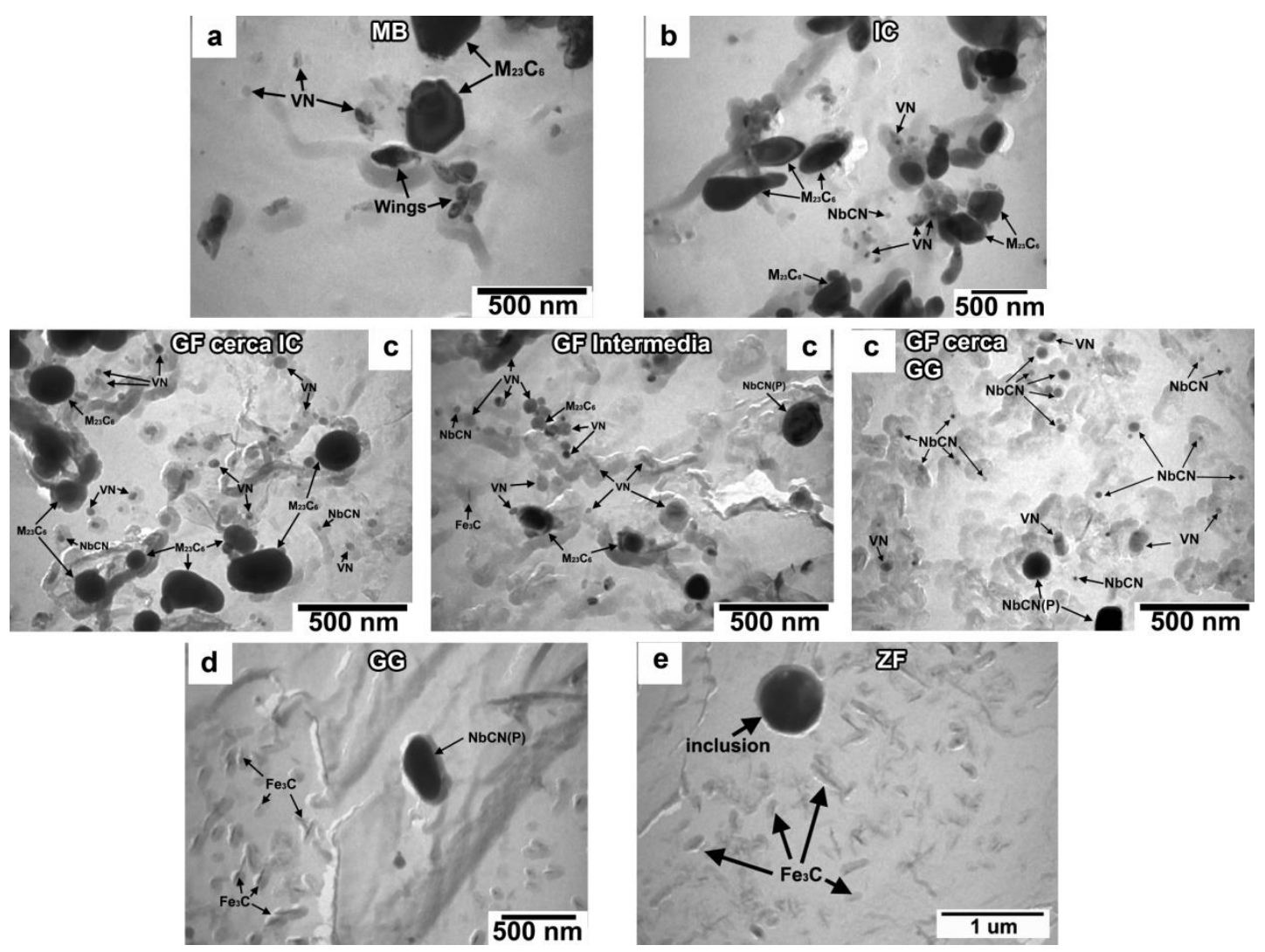

Figura 3: Micrografías TEM (réplicas extractivas de carbono) de las distintas zonas de soldadura por pasada simple: (a) MB, (b) ZACIC, (c) ZACGF, (d) ZACGG, (e) ZF.

En la Figura 4 se muestra en forma esquemática un resumen de los distintos precipitados encontrados en las distintas zonas del cordón de soldadura. Los números indican el orden en el cual los precipitados aparecen en las distintas zonas teniendo en cuenta la fracción de volumen estimado.

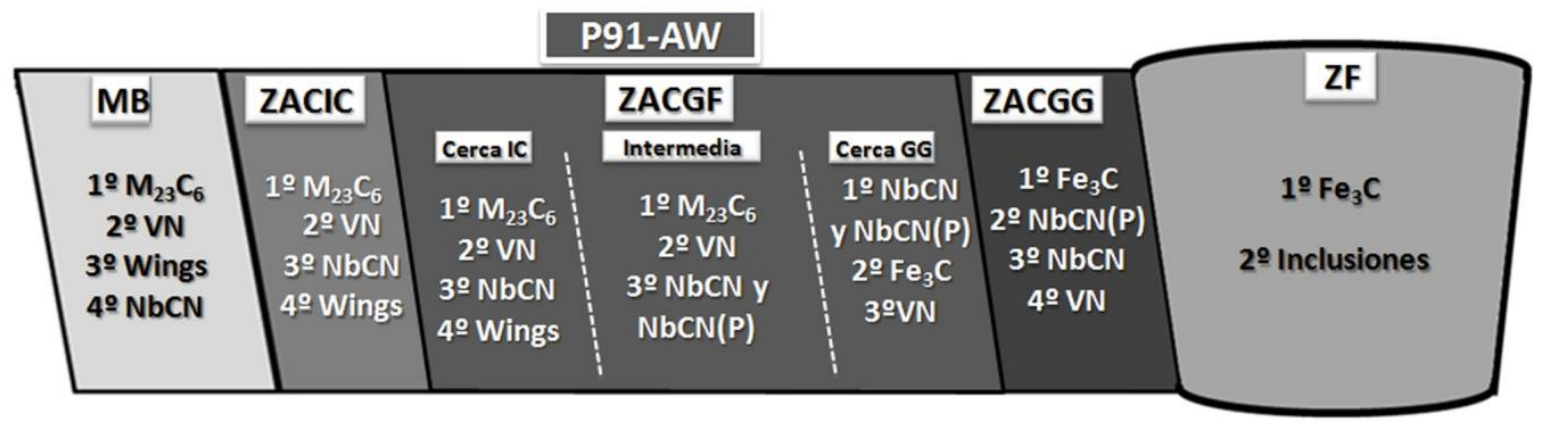

Figura 4: Esquema indicando los precipitados identificados distintas zonas de soldadura por pasada simple.

Como se puede observar en el esquema de la Figura 4, el precipitado mayoritario desde el MB hasta la ZACGF intermedia fue el carburo $\mathrm{M}_{23} \mathrm{C}_{6}$ y el MX mayoritario el VN. En la ZACGF cercana a la ZACGG los $\mathrm{M}_{23} \mathrm{C}_{6}$ desaparecen y el MX mayoritario es el NbCN. Se observaron $\mathrm{NbCN}(\mathrm{P})$ (P: primarios) desde la ZACGF Intermedia hasta la ZACGG. Los $\mathrm{NbCN}(\mathrm{P})$ son insolubles a las Tp alcanzadas en esas zonas y probablemente también podrían estar presentes en las zonas que alcanzan Tp más bajas y en el MB, pero es difícil encontrarlos en esas zonas debido a que quedan enmascarados por la gran cantidad de carburos $\mathrm{M}_{23} \mathrm{C}_{6}$ dado que tienen la misma morfología y tamaño [9]. A partir de la ZACGF cercana GG se observó $\mathrm{Fe}_{3} \mathrm{C}$, precipitado mayoritario en la ZACGG y en la ZF. La presencia de $\mathrm{Fe}_{3} \mathrm{C}$ se atribuye al autorevenido de la martensita lo cual es inevitable debido a la alta temperatura $\mathrm{M}_{\mathrm{s}}$ que presentan los aceros de bajo carbono [10].

En la Figura 5, se muestra el porcentaje $\mathrm{P}$ del área cubierta por los precipitados en las distintas subzonas de la ZAC comparado con el MB. Se midió el tamaño de los precipitados en 20 micrografías TEM de 
cada zona. El porcentaje de área cubierta por los precipitados en cada micrografía fue graficado con un punto verde para cada sub-zona de la ZAC y del MB. Los puntos rojos son los valores medios obtenidos promediando los puntos verdes.

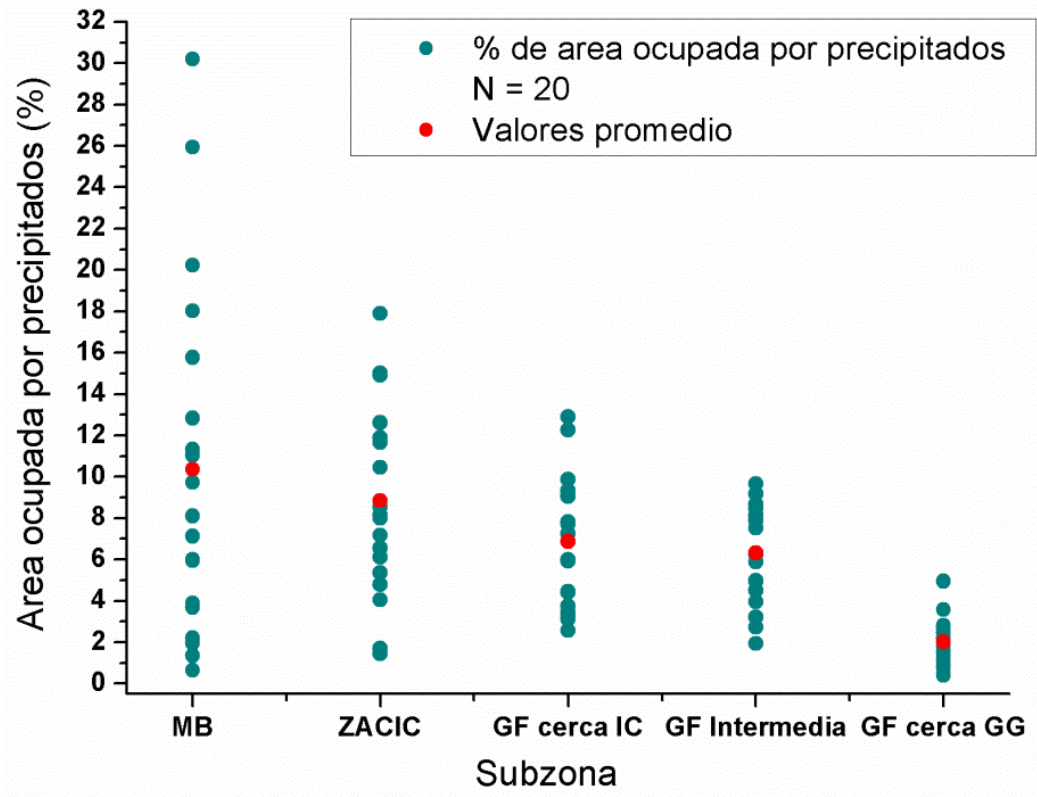

Figura 5: Porcentaje de área cubierta por precipitados en la ZAC y en el MB.

El MB es la zona que no fue afectada durante el proceso de soldadura, por lo tanto, su microestructura coincide con la del material en la condición de suministro. La Figura 6 muestra una estructura típica de la martensita revenida. Se observan precipitados en los ex bordes de grano austeníticos, en los bordes de listones y en el interior de los mismos. Resulta difícil observar a los carburos más finos debido a la alta densidad de dislocaciones.

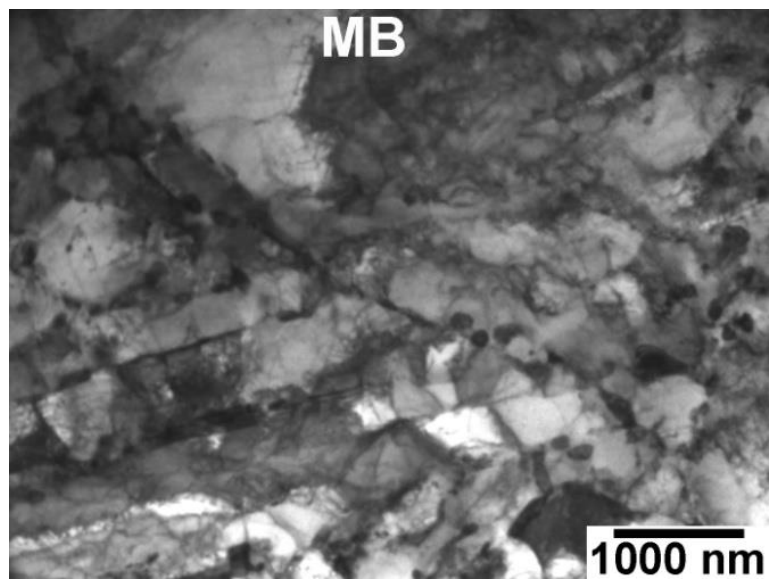

Figura 6: Micrografía TEM (lámina delgada) del MB.

En la Figura 7 se muestran las zonas de la ZAC de donde se extrajeron las muestras FIB señaladas con improntas. 


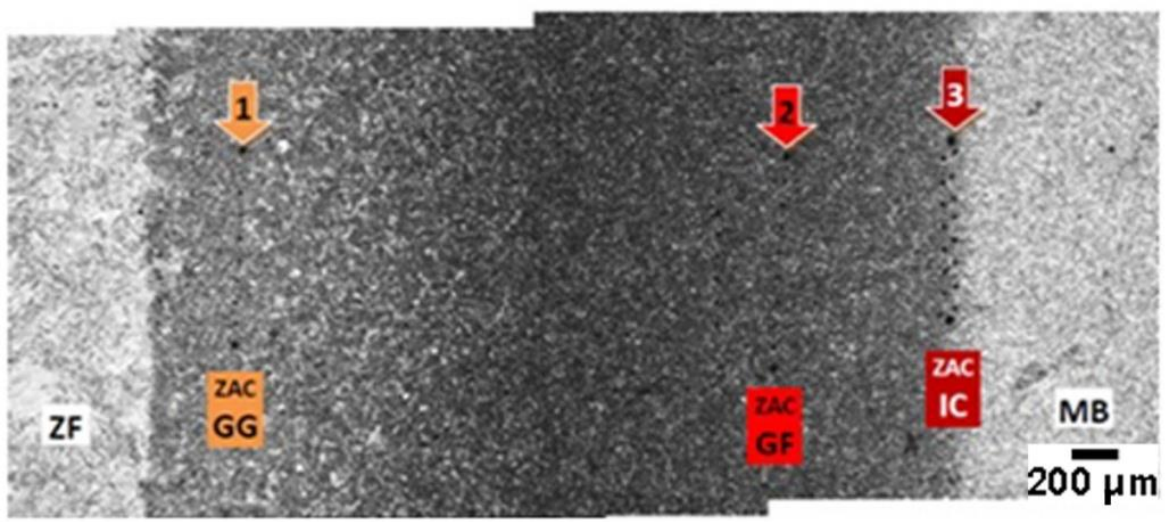

Figura 7: Micrografía óptica de las zonas de la ZAC de donde fueron extraídas las muestras mediante FIB.

En la Figura 8 se muestran micrografías SEM de las zonas seleccionadas para estudiar la ZACIC (Figura 8.a), la ZACGF (Figura 8.b) y la ZACGG (Figura 8.c). En las micrografías se observa el recubrimiento de platino que se utiliza para proteger la superficie de la muestra antes de realizar la lámina empleando el FIB.
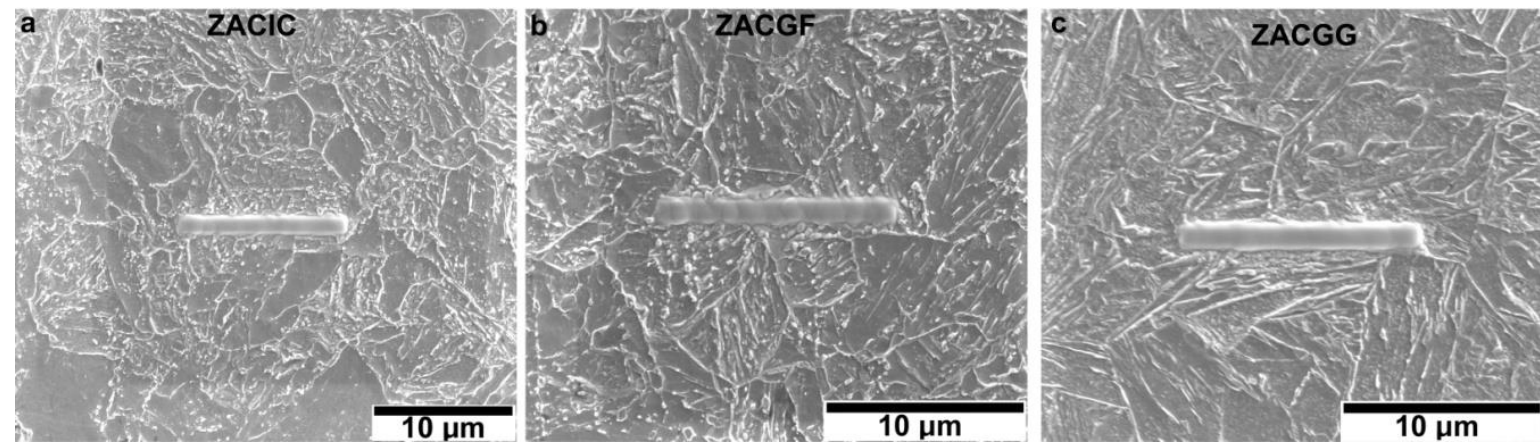

Figura 8: Micrografías SEM donde se indica, mediante el recubrimiento de Pt, las zonas donde se realizó la extracción de las láminas.

En las ZACIC y ZACGF se encontró austenita retenida alrededor de algunos precipitados $\mathrm{M}_{23} \mathrm{C}_{6}$ como se muestra en las micrografías de la Figura 9. 

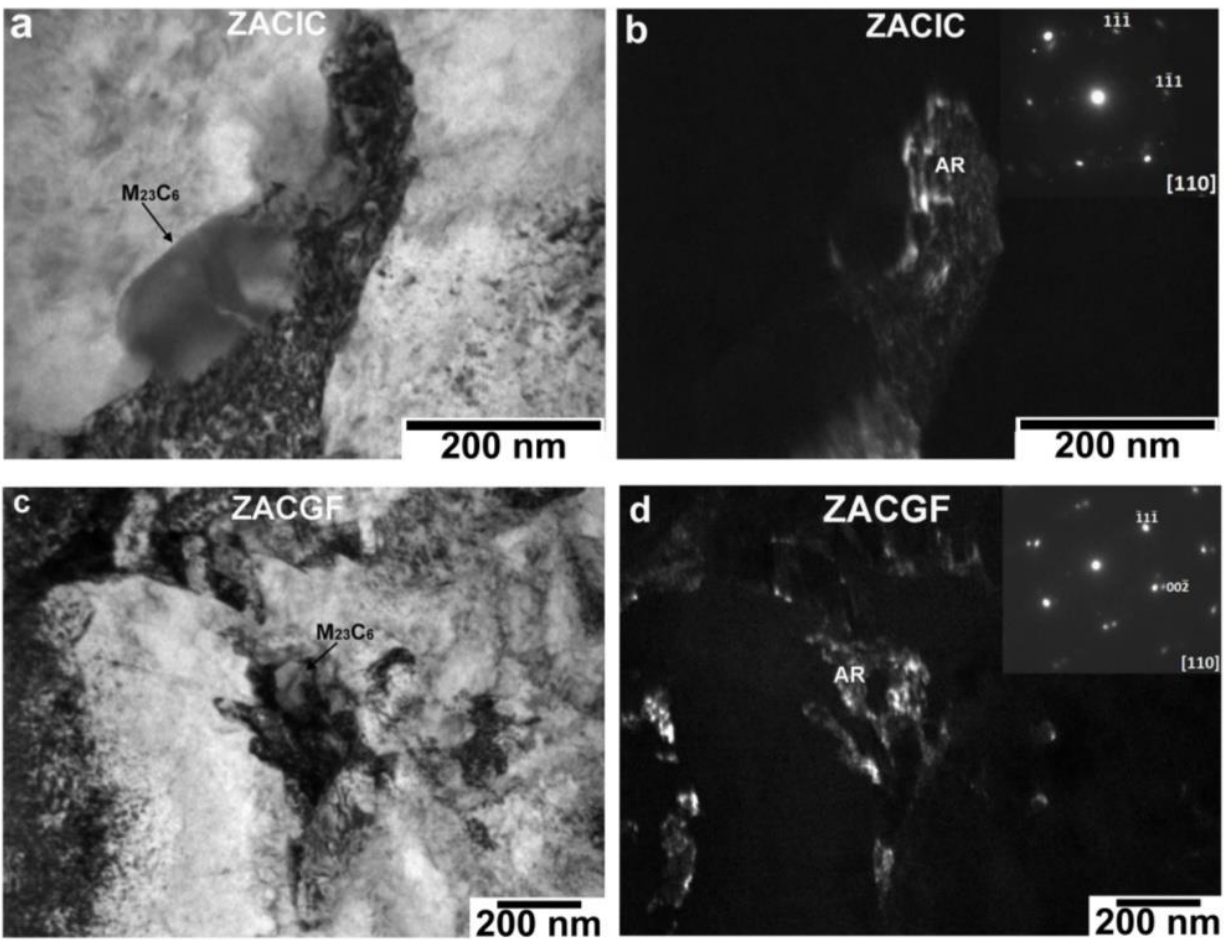

Figura 9: Micrografías TEM (láminas FIB). ZACIC: (a) Campo claro, (b) Campo oscuro y diagrama de difracción de electrones (D) en el eje de zona $\mathrm{z}=$ [110]. ZACGF: (c) Campo claro, (d) Campo oscuro y D en $\mathrm{z}=$ [110]. AR: austenita retenida.

En la ZACGG y ZF se pudo observar austenita retenida formando una película continua a lo largo de los ex bordes de grano austeníticos y en los bordes de listones de la martensita como se muestra en micrografías de la Figura 10.
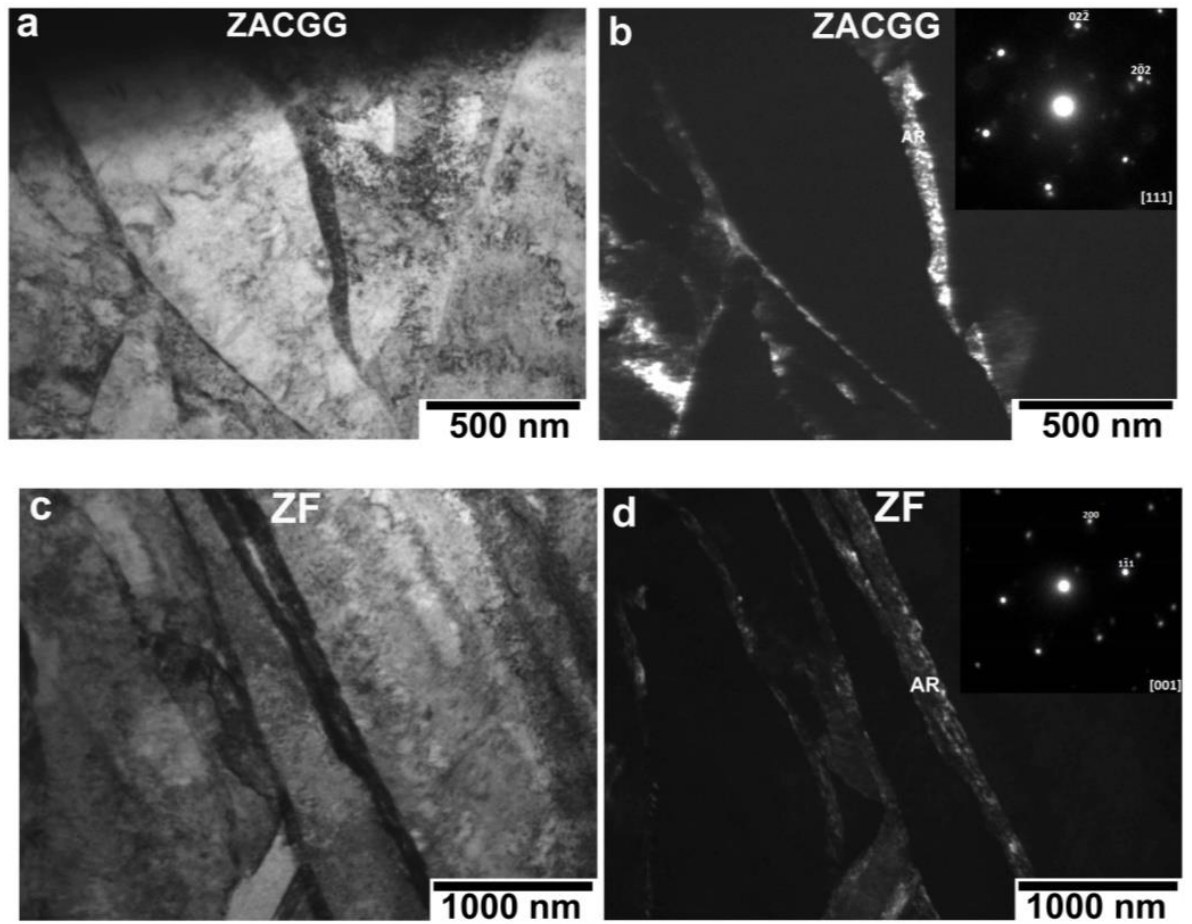

Figura 10: Micrografias TEM. ZACGG (lámina FIB): (a) Campo claro, (b) Campo oscuro y D en z = [111]. ZF (lámina delgada): (c) Campo claro; (d) Campo oscuro y D en z = [110]. 


\section{DISCUSIÓN}

Mediante cálculos termodinámicos se obtuvo, para la composición del acero del presente trabajo, la fracción de precipitados presentes a cada temperatura por encima de $780{ }^{\circ} \mathrm{C}$ (Figura 11). Cuando el acero es calentado/enfriado a altas velocidades tales como las presentes en las soldaduras, la disolución de los precipitados es diferida a más altas temperaturas que las predichas por las condiciones de equilibrio, pero la secuencia de disolución se mantiene. Los $\mathrm{M}_{23} \mathrm{C}_{6}$ son los precipitados mayoritarios seguidos por los $\mathrm{VN}$ en las zonas del material sometidas a temperaturas por debajo de las alcanzadas en la ZACGF cercana a la ZACGG. En esta última zona, ya no se observan $\mathrm{M}_{23} \mathrm{C}_{6}$ y los $\mathrm{NbCN}$ pasan a ser los precipitados mayoritarios superando en cantidad a los VN. En la ZF se disolvieron todos los precipitados presentes en el MB.

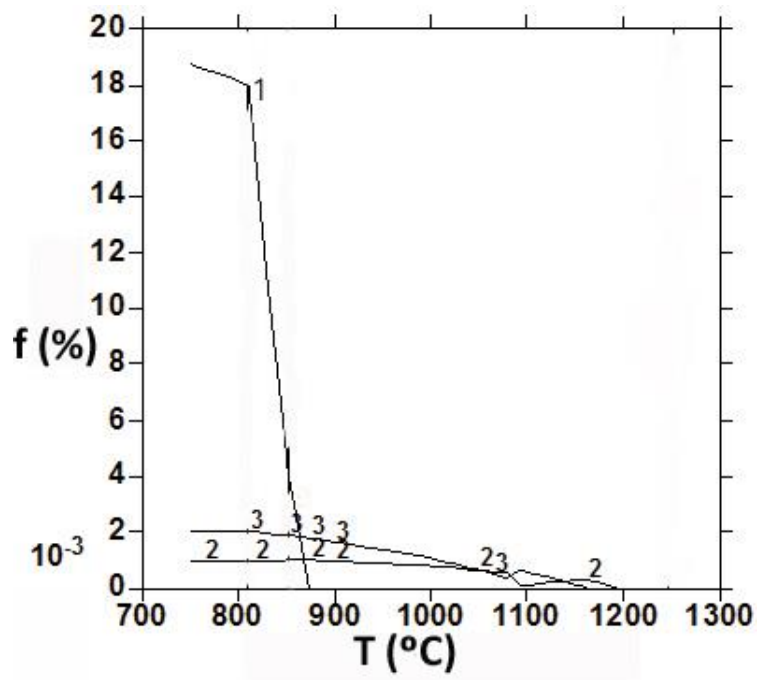

Figura 11: Cálculos teóricos de la evolución con la temperatura de los $\mathrm{M}_{23} \mathrm{C}_{6}$ (1), $\mathrm{NbCN}$ (2) y VN (3).

En las zonas que fueron sometidas a las más altas temperaturas pico y mayores velocidades de calentamiento/enfriamiento durante el proceso de soldadura, la ZF y la ZACGG, se encontró austenita retenida mayoritariamente en los bordes de los listones de martensita. Varios autores [11-13] reportaron austenita retenida con una morfología de película alargada sobre los bordes de listones. En particular, HURTADONOREÑA et al. [11], la austenita retenida fue encontrada en un acero P91 similar al del presente trabajo, normalizado a $1050^{\circ} \mathrm{C}$ por 30 minutos y enfriado al aire.

SHTANSKY et al [14] estudiaron la formación de austenita y disolución de carburos en una aleación $\mathrm{Fe}-8,2 \mathrm{Cr}-0,2 \mathrm{C}$. Partiendo de una matriz completamente ferrítica con carburos esferoidizados realizaron tratamientos térmicos que consistieron en mantener la temperatura entre 850 y $1150{ }^{\circ} \mathrm{C}$ entre 1 y 100 segundos seguidos por un templado en salmuera. Estos autores detectaron la formación de austenita mediante TEM después de 20,8 y 3 segundos a 850,870 y $900{ }^{\circ} \mathrm{C}$, respectivamente, hecho que muestra que la formación de esta fase es más rápida cuanto más alta sea la temperatura alcanzada. Observaron dos modos de formación de austenita en muestras tratadas a $870{ }^{\circ} \mathrm{C}$ en 10 segundos: el primero en los puntos triples de los bordes de grano en la vecindad con los carburos (detectaron fase $\gamma$ que transforma a $\alpha$ durante el templado) y el segundo directamente alrededor de algunos de los carburos (austenita retenida, Figura 12.a). El hecho que la austenita fuera retenida con el templado los llevó a sugerir que el material tenía un alto contenido de $\mathrm{C}$ y $\mathrm{Cr}$ en esas zonas lo cual coincide con los resultados de HOWELL et al. [15] quienes afirman que la estabilización de la austenita puede depender de la presencia de $\mathrm{C}$ y un nivel significativo de un fuerte formador de carburos. Cuando SHTANSKY et al [14] observaron muestras que habían estado a $870{ }^{\circ} \mathrm{C}$ durante 100 segundos, a $1000{ }^{\circ} \mathrm{C}$ durante 20 segundos y a $1150^{\circ} \mathrm{C}$ durante 10 segundos, encontraron una capa de ferrita alrededor de los carburos que se estaban disolviendo (Figura 12.b). También observaron regiones ferríticas con una extensión entre 0,5 y $1 \mu \mathrm{m}$ después de una completa disolución de carburos en la región que antes ocupaban los carburos (Figura 12.c). Estos autores pudieron distinguir la ferrita de la martensita por la diferencia en la densidad de dislocaciones de ambas fases (mucho más baja en la ferrita). En el presente trabajo, se observó austenita retenida en contacto con algunos $\mathrm{M}_{23} \mathrm{C}_{6}$ de la ZACIC y ZACGF lo cual coincide con lo encontrado por SHTANSKY et al [14] cuando estudiaron el material tratado a $870{ }^{\circ} \mathrm{C}$ durante 10 segundos. Las regiones de grano fino analizadas en el presente trabajo corresponden a las posiciones 2 y 3 indicadas con flechas en la Figura 7 , lo que significa que existen zonas de grano fino no estudiadas, que alcanzaron Tp más altas, las cuales permanecieron durante mayores tiempos a temperaturas por encima de $A_{c 1}\left(A_{c 1}=880{ }^{\circ} \mathrm{C}\right.$ para este 
material [16]), por lo tanto, en un futuro trabajo se piensa analizar estas zonas extrayendo láminas delgadas mediante FIB. Teniendo en cuenta que los registros de la evolución de la temperatura realizados con termocuplas durante el proceso de soldadura se obtuvo que el tiempo de permanencia a temperaturas mayores que $\mathrm{A}_{\mathrm{c} 1}$ en las regiones que alcanzaron Tp de 1055 y $1264{ }^{\circ} \mathrm{C}$ fueron de 2 y 15 segundos, respectivamente. Entonces, para las Tp de la ZACGF cercana a la GG (alrededor de los $1100{ }^{\circ} \mathrm{C}$ según se observa en el esquema de la Figura 1) el tiempo de permanencia estaría entre los 2 y 15 segundos. Luego, los tiempos de permanencia en fase austenítica para soldaduras reales serían inferiores a los tiempos de permanencia para los cuales SHTANSKY et al [14] observaron ferrita alrededor o reemplazando a los carburos $\mathrm{M}_{23} \mathrm{C}_{6}$, por lo tanto, esta fase podría no estar presente en las soldaduras reales.

Por otro lado, este trabajo se llevó a cabo en la condición "as welding" por lo tanto, es necesario continuar los estudios en muestras que hayan recibido un tratamiento térmico de post-soldadura para ver en qué transforma la austenita retenida con el efecto del revenido.
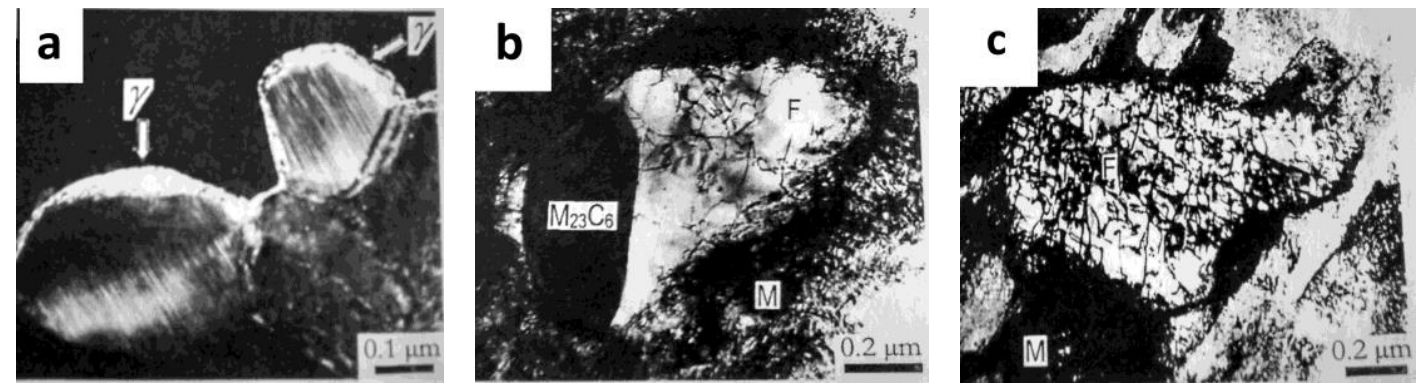

Figura 12: Aleación $\mathrm{Fe}-8,2 \mathrm{Cr}-0,2 \mathrm{C}$. (a) $870^{\circ} \mathrm{C}$ por $10 \mathrm{~s}$, presencia de austenita retenida $\left(\gamma\right.$ ); (b) $870^{\circ} \mathrm{C}$ por $100 \mathrm{~s}$, disolución parcial del $\mathrm{M}_{23} \mathrm{C}_{6}$; (c) $870{ }^{\circ} \mathrm{C}$ por 100 s, disolución completa del carburo. F: ferrita, M: martensita. [14].

\section{CONCLUSIONES}

Se realizó un estudio completo de la microestructura de las distintas zonas y sub-zonas de un cordón de soldadura por pasada simple del acero P91 en la condición "as welding" sobre una soldadura real. A pesar de las dificultades para extraer material de cada sub-zona de la ZAC, se pudo salvar este inconveniente realizando réplicas de carbono (enmascarando todas la zonas excepto la que estaba bajo estudio) y láminas (empleando un FIB) lo que permitió hacer un estudio mediante microscopía electrónica de transmisión en soldaduras reales.

Se identificaron los precipitados presentes en cada sub-zona de la ZAC y cómo la disolución del $\mathrm{M}_{23} \mathrm{C}_{6}$ afecta a la matriz que lo rodea. A bajas temperaturas pico y bajas velocidades de calentamiento/enfriamiento tales como las que caracterizan a las zonas de grano fino (ZACIC y ZACGF) se encontró austenita retenida en contacto de los carburos $\mathrm{M}_{23} \mathrm{C}_{6}$ parcialmente disueltos. Por otra parte, en las zonas con más altas temperaturas pico y altas velocidades de calentamiento/enfriamiento (ZACGG y ZF) se encontraron películas finas de austenita retenida en los ex bordes de grano austenítico y en los listones de martensita

\section{AGRADECIMIENTOS}

Al Dr. Daniel Vega de la División de Materia Condensada, GAYANN, Comisión Nacional de Energía Atómica por la asistencia con la técnica de Difracción de Rayos X, Sr. Gonzalo Zbihlei de la División Microscopía Electrónica, Gerencia Materiales, Comisión Nacional de Energía Atómica por a la asistencia con el TEM y al Sr. Oscar del Laboratorio de blancos, Gcia. GAYANN, Comisión Nacional de Energía Atómica por la asistencia con el depósito de carbono para realizar las réplicas. ALM y MIL agradecen el apoyo financiero de la Agencia Nacional de Ciencia y Tecnología de Argentina por medio del PICT 2014 No 2170. FS y MZ agradecen el aporte financiero de la Unión Europea en el marco del proyecto SUMA2 (IRSES Project $\mathrm{N}^{\circ}$ 318903).

\section{BIBLIOGRAFÍA}

[1] FUJITA, T. "Current progress in advanced high Cr ferritic steels for high-temperature applications", ISIJ International, v. 32, n. 2,pp. 175-181, 1992.

[2] MASUYAMA, F., "History of power plants and progress in heat resistant steels", ISIJ International, v. 41, n.6, pp. 612-625, 2001.

[3] KLUEH, L., "Elevated-temperature ferritic and martensítica steels and their application to future nuclear 
reactors", International Materials Review, v. 50, n. 5, pp. 287-310, 2005.

[4] FAZIO, C., ALAMO, A., ALMAZOUZI, A., et al., "European cross cutting research on structural materials for Generation IV and transmutation systems", Journal of Nuclear Materials, v. 392, n. 2, pp. 316-323, 2009.

[5] CERJAK, H., MAYR, P. “Creep strength of welded joints of ferritic steels", In: Abe, F., Torsten-Ulf, K., Viswanathan, R., Creep-resistent steel, 1 ed., chapter 17, Cornwall, England, Woodhead Publishing Limited, 2008.

[6] OTOGURO, Y., MATSUBARA, M., ITOH, I., et al., "Creep rupture strength of heat affected zone for 9Cr ferritic heat resisting steels", Nuclear Engineering and Design, v. 196,pp. 51-61,2000.

[7] ABE, R., "R \& D of advanced ferritic steels for $650{ }^{\circ} \mathrm{C}$ USC boilers", In: Proceedings International Workshop on the innovative structural materials for infrastructure in 21 st century, pp. 119-129, Tsukuba, Jan. 2000.

[8] ZAVALETA, G., CICCO, H., MARRERO, J., et al., "Evolution of precipitated phases during prolonged tempering in a $9 \% \mathrm{Cr} 1 \% \mathrm{MoVNb}$ ferritic-martensitic steel: Influence on creep performance", Materials Science and Engineering A, v. 528A, n. 12,pp. 4019-4029, 2011.

[9]ANDERSON, P., BELLGART, T., JONES, F.L., "Creep deformation in a modified 9Cr-1Mo steel, Materials Science Technology, v. 19, pp. 207-213, 2003.

[10] ABORN, R, "Low Carbon Martensite", Transactions of American Society for Metals, v. 48, pp. 51-57, 1956.

[11] HURTADO-NOREÑA, C., DANÓN, C., LUPPO, M. I., "Evolution of minor phases in a 9PctCr steel: Effect of tempering temperature and relation with hydrogen trapping", Metallurgical and Materials Transactions A, v. 46A, n. 9, pp. 3972-3988, 2015.

[12]SONG, M., SUN, C., FAN, Z., et al., "A roadmap for tailoring the strength and ductility of ferritic/martensitic T91 steel via thermo-mechanical treatment”, Acta Materialia, v. 112, pp. 361-377, 2016.

[13] SHIUE, R. K., LAN, K. C., CHEN, C., "Toughness and austenite stability of modified 9Cr-1Mo welds after tempering", Materials Science and Engineering A, v. 287, pp. 10-16, 2000.

[14] SHTANSKY, D., V., NAKAI, K., OHMORI, Y., "Formation of austenite and dissolution of carbides in Fe-8.2Cr-C alloys", Z. Metallkd, v. 90, n. 1, pp. 25-37, 1999.

[15] HOWELL, P. R., "The crystallography of the austenite-ferrite/carbide transformation in $\mathrm{Fe}-\mathrm{Cr}-\mathrm{C}$ alloys", Metallurgical Transactions A, v. 10, pp. 1213-22, 1979.

[16] Tesis MARZOCCA, A. L., Caracterización de precipitados presentes en un acero ASTM A335 Gr P91 soldado por proceso FCAW, Tesis de M.Sc., UNSAM/CNEA, Buenos Aires, Bs. As., Argentina, 2014. 